\title{
PERFIL E REABILITAÇÃO DE PACIENTES COM TENDINOPATIA NO SETOR DE FISIOTERAPIA DE UMA CLÍNICA-ESCOLA
}

Heloísa Paes Lima ${ }^{1}$, Eduardo Pizzo Junior ${ }^{1}$, Jaqueline Santos Silva ${ }^{1,2}$, Bruno Cézar Rotoly ${ }^{1,3}$, Gabriela Carrion Caldeira Ribeiro ${ }^{1}$, Natanael Pereira Batista ${ }^{1}$, Caio Ferreira Ripper ${ }^{1}$, Henrique Martins Ungri $^{1}$, Carlos Iván Mesa Castrillón ${ }^{1,2}$, Jayme Netto Junior ${ }^{1,4}$, Carlos Marcelo Pastre ${ }^{1,4}$

Universidade Estadual Paulista “Júlio de Mesquita Filho" - UNESP, Curso de Fisioterapia, 1,2 Mestrado em Fisioterapia,

${ }^{1,3}$ Especialização em Fisioterapia Desportiva, ${ }^{1,4}$ Professor Doutor Departamento de Fisioterapia, Laboratório de Fisioterapia Desportiva-LAFIDE. Presidente Prudente, SP. E-mail: pastre@fct.unesp.br

\section{RESUMO}

Caracterizar o perfil de lesão de uma população específica e a partir de então desenvolver protocolos de tratamentos específicos. Foram analisados prontuários de 40 pacientes com diagnóstico de tendinopatia durante o período de 2009 a 2013, destacando-se as variáveis quanto à modalidade praticada, sexo, idade e local da lesão. A modalidade com maior incidência encontrada foi o atletismo com $55 \%$ dos pacientes. A maioria dos casos se apresentou em pessoas do sexo masculino, sendo $60 \%$ com idade menor que 23 anos. Houve prevalência de $67,5 \%$ das lesões no membro inferior. Há um grande número de lesões recidivas, talvez em razão do considerável de altas por abandono. Nota-se que o perfil dos pacientes reabilitados com tendinopatia, é caracterizado predominantemente por mecanismos de lesão por contato direto, com alto índice de desistência ao tratamento, maior incidência nos membros inferiores e maior número de casos em praticantes de atletismo.

Palavras chave: Tendinopatia, reabilitação, lesões em atletas, tendões, patologia.

\section{PROFILE AND REHABILITATION OF PATIENTS WITH TENDINOPATHY IN HIGH-SCHOOOL CLINIC OF PHYSICALTHERAPY}

\begin{abstract}
Characterizing the damage profile of a specific population and from then develop specific treatment protocols. 40 patient records were analyzed diagnosed with tendinopathy during the period 2009-2013, highlighting the variables on the sport practiced, gender, age and place of injury. The mode with highest incidence was found athletics with $55 \%$ of patients. Most cases presented in males, $60 \%$ younger than 23 years. There was a prevalence of $67.5 \%$ of the lesions in the lower limb. There are a lot of relapses injuries, perhaps in considerable reason for high abandonment. Note that the profile of patients rehabilitated with tendinopathy, it is predominantly characterized by direct contact injury mechanisms, high dropout rate to treatment, a higher incidence in the lower limbs and a higher number of cases in track and field athletes.
\end{abstract}

Keywords: Tendinopathy, rehabilitation, athetic injuries, tendons, patology. 


\section{INTRODUÇÃO}

A tendinopatia é uma das principais causas de incapacidade em indivíduos fisicamente ativos e de atletas, causa dor e consequente diminuição do desempenho ${ }^{1-3}$. Sua causa pode se dar a partir de fatores intrínsecos (variações anatômicas, desequilíbrio, fraqueza, rigidez muscular, nutrição, idade, frouxidão ligamentar, doenças sistêmicas, perfusão vascular, excesso de peso) e extrínsecos (trabalho, carga física, excesso de uso, equipamentos inadequados, erros técnicos e condições ambientai), os quais contribuem para os processos patológicos ${ }^{4,5}$.

O mecanismo de lesão da tendinopatia é descrito na literatura em duas vertentes, teoria mecânica e vascular. Tais teorias, não são mutuamente exclusivas, podendo estar relacionadas com os fatores intrínsecos e extrínsecos já citados. Em resposta ao mecanismo vascular fatores como envelhecimento, desuso físico ou trauma podem gerar hipóxia tecidual, levando a diminuição da viabilidade celular na região do tendão, ocasionando uma ruptura ou uma lesão de menor intensidade ${ }^{5}$.

O tempo de cicatrização do tendão divide-se em três fases: inflamatória, reparação e remodelamento ${ }^{6}$. O tratamento mais recomendado para reabilitação da patologia em questão é o conservador, o qual tem por objetivo alterar o metabolismo do tendão, auxiliando sua cicatrização e alterando as propriedades estruturais e mecânicas ${ }^{7}$. Por outro lado, o tratamento cirúrgico é indicado para cerca de $25 \%$ dos pacientes os quais possuem sintomas permanentes ${ }^{3,8}$.

A partir do exposto, nota-se importância empírica de evidências científicas que demonstrem tratamentos eficientes para patologia em questão, tendo em vista os prejuízos e impactos que ocasionam para atletas e não atletas. Assim, o objetivo do presente estudo foi caracterizar o perfil de lesão de uma população específica e a partir de então desenvolver protocolos de tratamentos específicos.

\section{MÉTODOS}

A presente pesquisa caracteriza-se por um estudo observacional retrospectivo, com fonte de dados baseada em prontuários de pacientes atendidos na clinica-escola, no setor de fisioterapia em medicina desportiva. Foram registradas informações do período de janeiro de 2009 a dezembro de 2013, sendo a triagem realizada por quatro fisioterapeutas entre os meses de julho e agosto de 2014, com dupla checagem para concordância entre todas as informações.

A população estudada foi constituída por 40 pacientes com diagnóstico clínico de tendinopatia, sendo 10 do sexo feminino e 30 do sexo masculino, e houve a exclusão de apenas um prontuário devido à ausência de informações referentes ao tratamento fisioterapêutico realizado.

As variáveis analisadas foram referentes aos dados pessoais dos pacientes como sexo, idade, peso e estatura; além de informações sobre o ano em que foi realizado o atendimento, modalidade do atleta, detalhes sobre a lesão segundo local acometido, mecanismo.

Em relação aos dados pessoais, as medidas de tendência central e variabilidade da idade, peso e estatura dos participantes são apresentadas por sexo na tabela 1.

Tabela 1. Medidas descritivas dos participantes da pesquisa segundo o sexo

\begin{tabular}{|c|c|c|c|}
\hline Sexo & \multicolumn{3}{|c|}{ Variáveis } \\
\hline & Idade (anos) & Peso (kg) & Estatura (m.) \\
\hline Feminino & $24,7 \pm 11,4$ & $60,6 \pm 2,95$ & $1,64 \pm 0,6$ \\
Masculino & $24,36 \pm 7,04$ & $72,23 \pm 9,76$ & $1,78 \pm 0,82$ \\
\hline
\end{tabular}


Em relação a variável ano, houve a divisão referente aos anos de atendimento informados nos prontuários, variando de 2009 a 2013. A variável modalidade apresentou incidência em diversos esportes, como atletismo, basquetebol, voleibol, futsal, musculação e lesão doméstica; e, a partir dessa verificação, foi dividida entre individual ou coletivo.

No que se refere à lesão, o local acometido foi referido especificamente conforme os locais anatômicos (joelho, tendão de Aquiles, tendão do supra-espinhal, músculo bíceps femoral, entre outros) e, em seguida, dividido em dois grupos como membros superiores ou membros inferiores. O mecanismo de lesão foi dividido em sem contato ou overuse.

Foi utilizado para análise dos dados o teste de GOODMAN ${ }^{9,10}$ para verificar a comparação de duas ou mais amostras, avaliando contrastes entre e dentro de populações multinominais, e o teste de Odds Ratio para verificar a razão de chance de associação por grupo com intervalo de confiança de $95 \%$. Todas as conclusões foram discutidas para $5 \%$ de significância estatística.

O estudo foi submetido ao Comitê de Ética em Pesquisa da Faculdade de Ciências e Tecnologia (FCT-UNESP), através do protocolo de número CAAE: 40628715.1.0000.5402.

\section{RESULTADOS}

A figura 1 representa a incidência de lesão em relação as modalidades acometidas. Nota-se que o atletismo foi a modalidade que apresentou a maior incidência de tendinopatia, sua ocorrência foi de cerca de $55 \%$ de todos os casos. O futsal foi a segunda modalidade com maior número de casos, com $15 \%$ do total observado, entretanto se comparado a modalidade anterior ele possui cerca de 3,5 vezes menos pacientes. O voleibol possui $10 \%$ dos pacientes acometidos pela tendinopatia, sendo assim, o terceiro com maior incidência dentre as modalidades analisadas no estudo. A modalidade com o quarto maior índice de lesão é o basquetebol, com $8 \%$ dos pacientes com a lesão. A musculação e as lesões domésticas são pouco encontradas, ficando assim em segundo lugar dentre as observadas com o menor número de casos encontrados, cada uma delas com $5 \%$ do total. A modalidade com a menor ocorrência dessa patologia foi a natação, com $3 \%$ do total de casos observados.

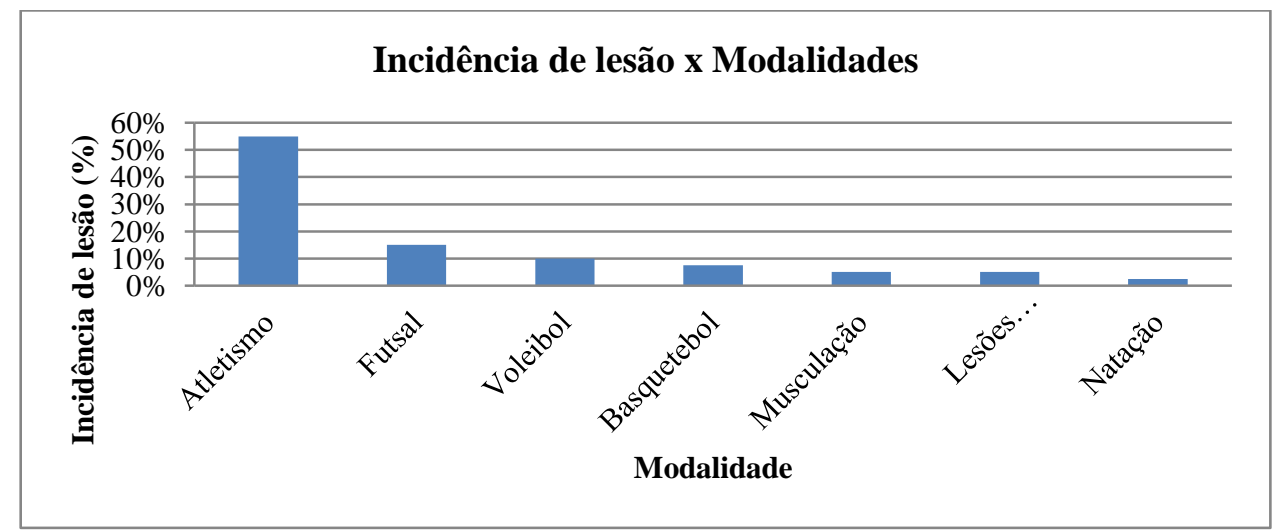

Figura 01. Incidência da quantidade de lesões em relação às modalidades do atleta.

Na tabela 2, observou-se que em relação ao sexo, a maioria das altas por aptidão alcançada foi de $70 \%$ das altas, encontrada no sexo feminino, enquanto que em relação as altas por abandono, a maior incidência foi no sexo masculino, com $46,7 \%$ do total de altas, entretanto, vale ressaltar que dentre os prontuários analisados, 10 eram de mulheres e 30 de homens. Quando observado a idade, encontra-se que nos adultos a quantidade de altas por aptidão alcançada e por abandono foi a mesma, sendo assim, 8 pacientes em cada tipo; já nos pacientes abaixo de 23 anos foi encontrado um maior número de altas por aptidão alcançada com 15 casos, representando assim $62,5 \%$ das altas nessa idade, 0 total de pacientes em cada idade foi de 24 nos que tem menos de 23 anos e 16 nos adultos. 
Ainda na tabela 1, percebe-se que em relação as modalidades, o maior número de altas por aptidão alcançada foi encontrado em esportes coletivos $(61,5 \%)$, no entanto nas altas por abandono o maior índice foi encontrado em esportes individuais $(44,4 \%)$; do total de pacientes acometido 27 são de modalidades individuais e 13 de modalidades coletivas. Em relação ao local da lesão, encontra-se maior número de altas por aptidão alcançada nas lesões de membro superior $(61,5)$, desta maneira nas altas por abandono o maior índice foi encontrado nas lesões de membro inferior (44,4\%); do total de pacientes com lesão, 27 possuem lesão no membro inferior e 13 no membro superior. Em relação as lesões recidivas, são encontrados menor índice de altas por aptidão alcançada em pacientes em que a lesão foi ausente $(57,1 \%)$, já nas altas por abandono o menor índice foi encontrado em pacientes com presença de lesões $(41,7 \%)$; o número total de pacientes com lesão recidiva ausente foi e 28 , enquanto eu o número total de pacientes com lesão recidiva presente foi 12 .

Tabela 02. Distribuição de frequências, absoluta e relativa (\%), das altas segundo as variáveis analisadas, respeitando o intervalo de confiança de $95 \%$.

\begin{tabular}{|c|c|c|c|c|c|}
\hline \multirow{2}{*}{$\begin{array}{l}\text { Variáveis } \\
\text { Analisadas }\end{array}$} & & \multicolumn{2}{|c|}{ Alta } & \multirow[t]{2}{*}{ Total } & \multirow[t]{2}{*}{$\mathrm{p}$ valor } \\
\hline & & $\begin{array}{c}\text { Aptidão } \\
\text { Alcançada }\end{array}$ & Abandono & & \\
\hline \multirow[t]{2}{*}{ Sexo } & Feminino & $7(70 \%)$ aA & $3(30 \%)$ aA & 10 & $p>0,05$ \\
\hline & Masculino & $16(53,3 \%)$ aA & $14(46,7 \%) \mathrm{aA}$ & 30 & $p>0,05$ \\
\hline \multirow[t]{2}{*}{ Idade } & Sub 23 & $15(62,5 \%) \mathrm{aA}$ & $9(37,5 \%)$ aA & 24 & $p>0,05$ \\
\hline & Adulto & $8(50 \%)$ aA & $8(50 \%)$ aA & 16 & $p>0,05$ \\
\hline \multirow[t]{2}{*}{ Modalidade } & Individual & $15(55,6 \%) \mathrm{aA}$ & $12(44,4 \%) \mathrm{aA}$ & 27 & $p>0,05$ \\
\hline & Coletivo & $8(61,5 \%)$ aA & $5(38, \% \%) a A$ & 13 & $p>0,05$ \\
\hline \multirow[t]{2}{*}{$\begin{array}{l}\text { Local da } \\
\text { lesão }\end{array}$} & $\begin{array}{l}\text { Membro } \\
\text { superior }\end{array}$ & $8(61,5 \%)$ aA & $5(38,5 \%)$ aA & 13 & $p>0,05$ \\
\hline & $\begin{array}{c}\text { Membro } \\
\text { inferior }\end{array}$ & $15(55,6 \%)$ aA & $12(44,4 \%) \mathrm{aA}$ & 27 & $p>0,05$ \\
\hline \multirow[t]{2}{*}{ Recidivas } & Ausente & $16(57,1 \%)$ aA & $12(42,9 \%)$ aA & 28 & $p>0,05$ \\
\hline & Presente & $7(58,3 \%)$ aA & $5(41,7 \%)$ aA & 12 & $p>0,05$ \\
\hline
\end{tabular}

\section{DISCUSSÃO}

De acordo com os achados deste estudo, pode-se observar que os homens foram os mais afetados, e que as modalidades de esporte coletivo apresentaram maior porcentagem de lesões, além de serem mais acometidos por lesões de grau II, e a inversão foi o mecanismo que provocou a maior porcentagem de entorse de tornozelo.

Segundo McKay et al. $45 \%$ das lesões no tornozelo ocorrem após salto com a superfície da quadra, o que corrobora com nosso estudo onde na modalidade vôlei e basquete observou-se apresentar entorse após o momento dos saltos ${ }^{11}$.

As evidências a partir dos achados apontam para uma conclusão de que sujeitos praticantes de modalidades coletivas estão mais expostos, sendo interessante realizar sistematicamente, principalmente em períodos de competição, trabalhos de prevenção que podem ser constituídos por exercícios de ganho de resistência, força, equilíbrio e estabilização.

$\mathrm{Na}$ análise de associação entre o mecanismo de lesão e contato direto e sem contato, foi possível observar que mecanismo sem contato foi mais frequente. As lesões sem contato são comuns devido às ações dinâmicas que englobam corrida, mudança de direção, rápida desaceleração, parada espontânea, movimento de torção e saltos ${ }^{12}$.

Destaca-se como limitação do estudo, o baixo número de prontuários analisados, o preenchimento incompleto dos prontuários e a ausência de informações relevantes, que 
dificultaram na conferência e interpretação dos mesmos. Sugere-se, que sejam incluídas nos prontuários avaliações padronizadas de dor, força e função, para melhor compreensão dos reais efeitos dos diferentes tipos de intervenções, bem como novos estudos com maior número de prontuários que investiguem as lesões de ligamento, para que se possa compreender o tratamento mais adequado e a real eficácia para recuperação funcional.

\section{CONCLUSÃO}

Nota-se que o perfil dos pacientes reabilitados com tendinopatia, atendidos em uma clinica-escola no setor de fisioterapia desportiva, é caracterizado predominantemente por mecanismo de lesão por contato direto, alto índice de desistência ao tratamento, maior incidência nos membros inferiores e maior número de casos em praticantes de atletismo.

\section{REFERÊNCIAS}

Maffulli N, Longo UG, Denaro V. Novel approaches for the management of tendinopathy. The Journal of bone and joint surgery American volume. 2010;92(15):2604-13.

Fahlstrom $\mathrm{M}$, Jonsson $\mathrm{P}$, Lorentzon $\mathrm{R}$, Alfredson $\mathrm{H}$. Chronic Achilles tendon pain treated with eccentric calf-muscle training. Knee surgery, sports traumatology, arthroscopy : official journal of the ESSKA. 2003;11(5):327-33. https://doi.org/10.1007/s00167-003-0418-z

Paavola M, Kannus P, Jarvinen TA, Khan K, Jozsa L, Jarvinen M. Achilles tendinopathy. The Journal of bone and joint surgery American volume. 2002;84-a(11):2062-76.

Fredberg U, Stengaard-Pedersen K. Chronic tendinopathy tissue pathology, pain mechanisms, and etiology with a special focus on inflammation. Scandinavian journal of medicine \& science in sports. 2008;18(1):3-15. https://doi.org/10.1111/j.1600-0838.2007.00746.x

Riley G. The pathogenesis of tendinopathy. A molecular perspective. Rheumatology (Oxford, England). 2004;43(2):131-42. https://doi.org/10.1093/rheumatology/keg448

Woo SL, Hildebrand K, Watanabe N, Fenwick JA, Papageorgiou CD, Wang JH. Tissue engineering of ligament and tendon healing. Clinical orthopaedics and related research. 1999(367 Suppl):S31223. https://doi.org/10.1097/00003086-199910001-00030

Wang JH, losifidis MI, Fu FH. Biomechanical basis for tendinopathy. Clinical orthopaedics and related research. 2006;443:320-32. https://doi.org/10.1097/01.blo.0000195927.81845.46

Maquirriain J, Ayerza M, Costa-Paz M, Muscolo DL. Endoscopic surgery in chronic achilles tendinopathies: A preliminary report. Arthroscopy : the journal of arthroscopic \& related surgery : official publication of the Arthroscopy Association of North America and the International Arthroscopy Association. 2002;18(3):298-303. https://doi.org/10.1053/jars.2002.30065b

Goodman LA. On Simultaneous Confidence Intervals for Multinomial Proportions. Technometrics. 1965;7(2):247-54. https://doi.org/10.1080/00401706.1965.10490252

Goodman LA. Simultaneous Confidence Intervals for Contrasts Among Multinomial Populations. The Annals of Mathematical Statistics. 1964;35(2):716-25. https://doi.org/10.1214/aoms/1177703569 
McKay GD, Goldie PA, Payne WR, Oakes BW. Ankle injuries in basketball: injury rate and risk factors. British journal of sports medicine. 2001;35(2):103-8. https://doi.org/10.1136/bjsm.35.2.103

Brito J, Soares J, Rebelo AN. Prevenção de lesões do ligamento cruzado anterior em futebolistas. Revista Brasileira de Medicina do Esporte. 2009;15:62-9. https://doi.org/10.1590/S1517$\underline{86922009000100014}$ 